\title{
Communication using Synchronization of Chaos in Semiconductor Lasers with optoelectronic feedback
}

\author{
S. Tang* , L. Illing ${ }^{\dagger}$, J. M. Liu*, H. D. I. Abarbanel ${ }^{* *}$ and M. B. Kennel ${ }^{\ddagger}$ \\ ${ }^{*}$ Department of Electrical Engineering, University of California, Los Angeles, Los Angeles, CA \\ 90095, USA \\ ${ }^{\dagger}$ Institute for Nonlinear Science and Department of Physics, University of California, San Diego, \\ La Jolla, CA 93093-0402, USA \\ ${ }^{* *}$ Institute for Nonlinear Science, Department of Physics and Marine Physical Laboratory at \\ Scripps Institution of Oceanography, University of California, San Diego, La Jolla, CA \\ 93093-0402, USA \\ ${ }^{\ddagger}$ Institute for Nonlinear Science, University of California, San Diego, La Jolla, CA 93093-0402,
} USA

\begin{abstract}
A communication scheme based on the synchronization of two chaotic semiconductor lasers is experimentally tested. The Chaos in the single-mode semiconductor lasers is generated by means of an optoelectronic feedback. Synchronization of the chaos is achieved by coupling a fraction of the transmitter's output power into the driving current of the receiver. We present experimental results on the route to chaos and the synchronization of $\mathrm{GHz}$ chaotic signals. We then test a proposed communication scheme by successfully transmitting messages.
\end{abstract}

\section{INTRODUCTION}

Communications using chaotic waveforms as "carriers" of information promise possible advantages over traditional communications strategies in the achievement of power efficiency gains and better use of broadband capability. Optical communication utilizing synchronized chaotic lasers with optoelectronic feedback has been demonstrated for quite slow data rates by using chaotic wavelength fluctuations [1]. The first high speed optical chaotic communication has been achieved using fiber ring lasers with a rare earth doped fiber segment as active element [2]. We found in our earlier work on such erbium doped fiber ring lasers [3] that, although observed bit rates up to $250 \mathrm{Mbits} / \mathrm{s}$ are interesting in principle, the change of the chaotic waveform in these types of lasers is slow compared to the time it takes the light to make one round trip in a standard laser composed of tens of meters of passive optical fiber. For practical applications, where bit rates of gigabits per second are desirable, one has to consider different active elements in the laser cavity, which give rise to fast chaos. In this paper we investigate semiconductor lasers with optoelectronic feedback. This is one way to generate high-frequency chaos and has in comparison to optical feedback the advantage of being phase insensitive. 


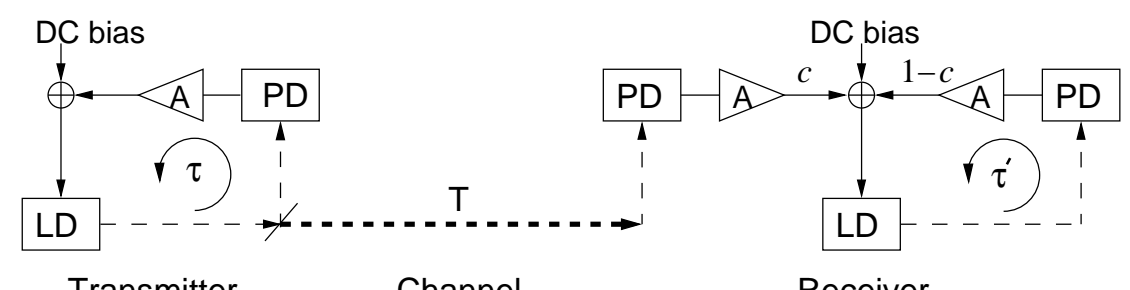

FIGURE 1. Schematic experimental setup for the synchronization of two chaotic semiconductor lasers with delayed optoelectronic feedback. LD: laser diode; PD: photodetector; A: amplifier; $\tau$ : feedback delay time; T: transmission time.

\section{CHAOS IN OPTOELECTRONIC FEEDBACK LASERS}

A schematic of our experimental setup for the investigation of semiconductor lasers with optoelectronic feedback can be seen in Fig. 1. In this section we are only concerned with the dynamics of the transmitter in Fig. 1. The laser diode is driven by a DC bias current and lases in a single mode. The output light is received and converted to a current by a photodetector. The current is subsequently amplified and added to the bias current, resulting in a delayed positive feedback. The delay time can be varied by changing the distance between the laser diode and the photodetector.

In our numerical investigations of the delay differential equations, which describe the dynamics of the optoelectronic feedback laser, we find a rich bifurcation diagram as the delay time and the feedback strength are varied $[4,5]$. In general chaotic regions are interspersed with periodic and quasi-periodic ones and multistability of different types of attractors, e.g. fixed points and limit cycles, are a common feature. We find that the optoelectronic feedback laser enters chaos through the quasi-periodic route. This agrees well with results from analytic bifurcation analyses of the delay differential equations modeling the system $[6,7]$.

In the experiments we use a single mode DFB laser diode with a wavelength of $1.3 \mu \mathrm{m}$ from Rockwell. The threshold of the laser is $34 \mathrm{~mA}$ and the laser is biased at $45.5 \mathrm{~mA}$. The light is converted to a current by a high-speed InGaAs photodetector (6 GHz bandwidth). The amplifier in the experiment is composed of two stages of Avantek SSF86 amplifiers (0.4 - $3 \mathrm{GHz}$ bandpass). The small signal gain of this twostage amplifier is about $60 \mathrm{~dB}$. The optical output detected by the photodetector is observed with an HP Agilent 54845A Infiniium oscilloscope with a $1.5 \mathrm{GHz}$ bandwidth and an up to $8 \mathrm{GSa} / \mathrm{s}$ sampling rate. The power spectrum is measured with an HP 8592A $\mathrm{RF}$ spectrum analyzer. The experiment is performed by changing the delay time through varying the distance between the laser diode and the photodetector.

Fig. 2 shows the experimentally observed time series of the laser output, the corresponding power spectra, and the corresponding Poincare section at different time delays. The Poincare section is obtained in a delay-embedding space using the experimental time series. The data shown in Figs. 2(a)-(c) were taken at measured delay times of $\tau \simeq 7.47 \mathrm{~ns}, 7.09 \mathrm{~ns}$ and $6.92 \mathrm{~ns}$, respectively. The time series in Figs. 2(a)-(c) show a clear transition from regular limit cycle oscillations to quasi-periodic oscillations with 

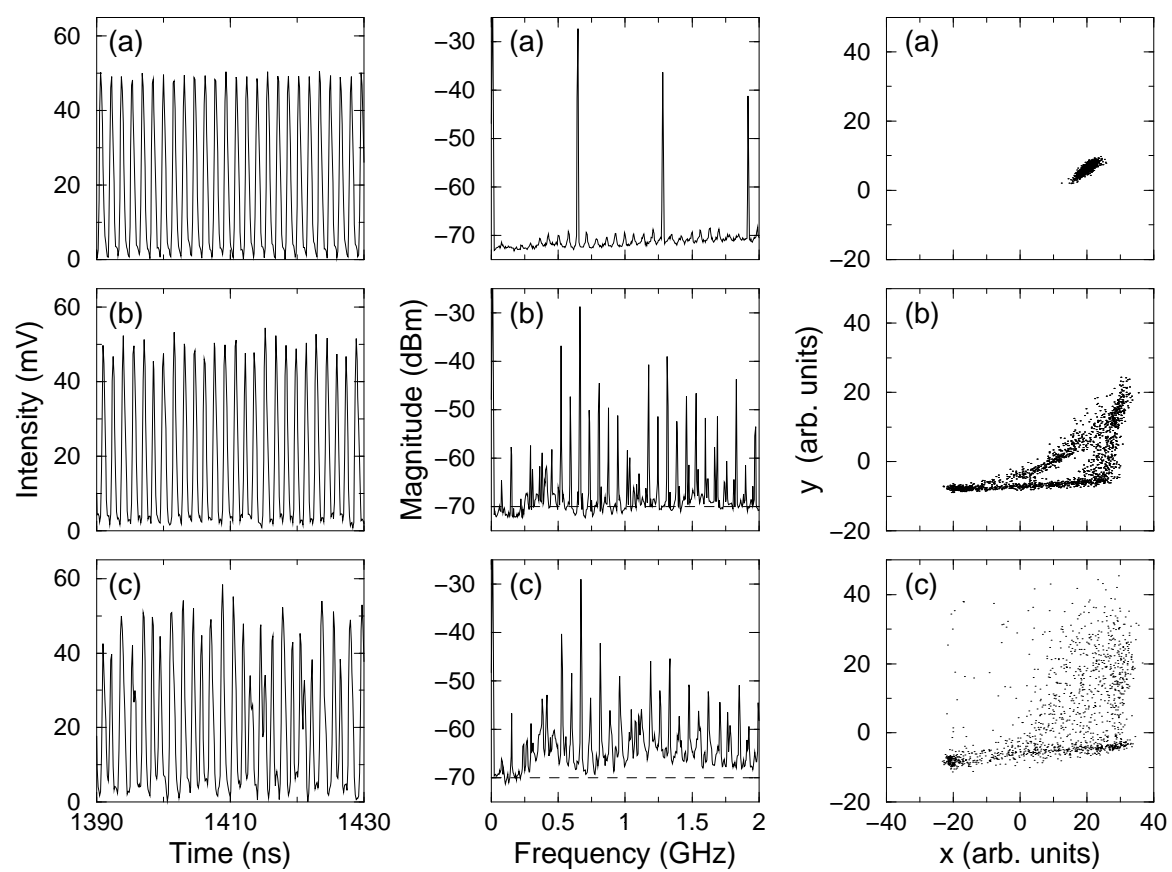

FIGURE 2. Experimental results: time series, power spectra and Poincaré sections at different delay times. From top to bottom: (a) limit cycle oscillations, at $\tau \simeq 7.47 \mathrm{~ns}$; (b) two-frequency quasi-periodicity, at $\tau \simeq 7.09 \mathrm{~ns}$; (c) chaos, at $\tau \simeq 6.92 \mathrm{~ns}$. The broadband background in (c) is much higher than in (b), indicated by the dashed reference lines at $-70 \mathrm{dBm}$.

intensity amplitudes modulated at a certain second frequency, and finally to a chaotic state where the intensities vary irregularly. As limited by the sampling rate of the oscilloscope, the pulses are under-sampled, resulting in large digitizing errors. To avoid the digitizing errors, the power spectra are measured with an RF spectrum analyzer. From the power spectra, it is clear that in Fig. 2(a) there is only one fundamental frequency at $f_{1} \simeq 650 \mathrm{MHz}$. The small ripples in the spectrum indicate that there is small instability in the laser. Then in Fig. 2(b), a second frequency at $f_{2} \simeq 140 \mathrm{MHz}$, which corresponds to the inverse of the delay time, is as significant as $f_{1}$. The two frequencies $f_{1}$ and $f_{2}$ are incommensurate and the system is in a two-frequency quasi-periodic oscillating state. Finally in Fig. 2(c), the spectrum is broadened as the system enters a chaotic pulsing state. Though some of the sharp spectral peaks remain in Fig. 2(c), the broadband background, a characteristic of chaos, is much higher in Fig. 2(c) than in Fig. 2(b), as indicated by the dashed reference lines at $-70 \mathrm{dBm}$. The big dot in the Poincaré section in Fig. 2(a) corresponds to regular oscillations. The size of the dot is an indication of the noise in the data. Despite the noise and the under-sampling the toroidal nature of the attractor for $\tau \simeq 7.09 \mathrm{~ns}$ can clearly be seen as a diffused closed curve in Fig. 2(b). In Fig. 2(c) the spread of the Poincaré plot indicates the transition to chaos.

Overall the numerical and experimental results show good agreement. The predicted quasi-periodic route to chaos is experimentally observed. 


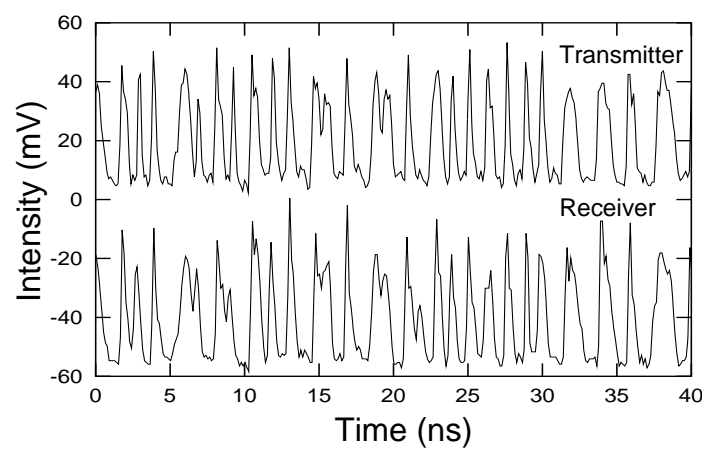

(a)

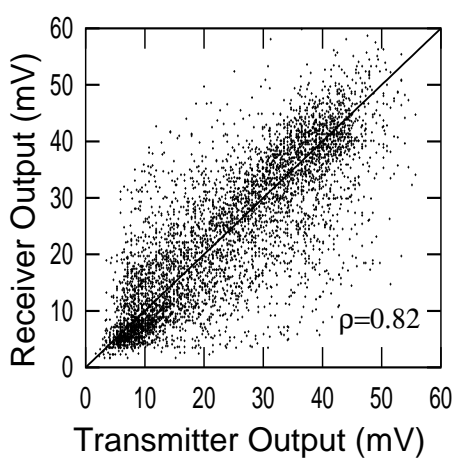

(b)

FIGURE 3. Experimental result of chaotic synchronization with c=0.6. (a) Time series of transmitter and receiver. (b) Correlation diagram of the transmitter output vs. the receiver output.

\section{CHAOS SYNCHRONIZATION}

The schematic setup of the synchronization experiment is shown in Fig. 1, where a fraction of the transmitter's chaotic output intensity is detected, amplified and coupled into the driving current of the receiver. The receiver is itself a delayed optoelectronic feedback laser. As in our work on the synchronization of doped fiber ring lasers $[8,9]$ we include the factor $c$, with $0 \leq c \leq 1$, to indicate the percentage of the total feedback signal in the receiver which is from the transmitter, while $1-c$ corresponds to the fraction from the receiver. When $c=1$ there is no feedback signal in the receiver and we call this open loop. When $c=0$ the transmitter and receiver are completely decoupled. We denote by $T$ the transmission time; the time associated with the propagation of the signal thru the open air 'channel' to the receiver system. When the transmitter and receiver are identical, a state where the dynamical variables of both are equal in time is a solution for the coupled system. We found numerically, that for $c$ roughly 0.4 or larger, this solution is stable and synchronization of the electric field and the carrier density in transmitter and receiver is accomplished both with and without spontaneous emission noise [5]. We furthermore showed in [5] that synchronization is most sensitive to parameter mismatch in the delay time, with small synchronization errors for mismatches of up to a few percent for any other parameter.

To achieve synchronization, the laser parameters are matched by carefully choosing a pair of lasers from the same batch with the closest characteristics and then fine-tuning their operating conditions. Fig. 3 shows the experimental results for synchronization with $c=0.6$, a configuration where the feedback loop in the receiver is closed. A comparison of the time series in Fig. 3(a) shows that they are almost identical. Note that the time series in Fig. 3(a) are shifted in time by the transmission time $T$ (see Fig. 1) to better show the synchronization. The correlation plot Fig. 3(b) is obtained by plotting the transmitter output vs. the receiver output. The data is distributed along the $45^{\circ}$ line, indicating identical synchronization. The quality of synchronization is limited by both the intrinsic laser noise and the finite bandwidth and sampling rate of the oscilloscope. We calculate the correlation coefficient $\rho$ of the transmitter and receiver 


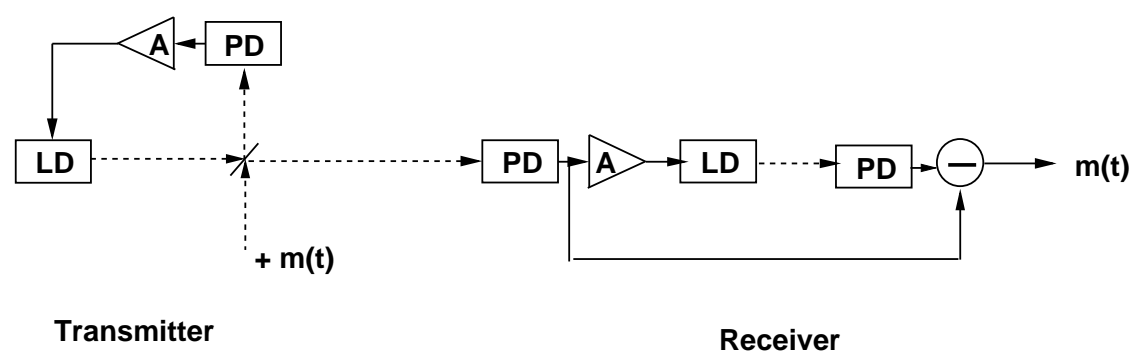

FIGURE 4. Schematic experimental setup for the chaotic communication scheme. LD: laser diode; PD: photodetector; A: amplifier; $\mathrm{m}(\mathrm{t})$ : message

output to quantify the quality of synchronization. For the data of Fig. 3 the correlation coefficient is $\rho=0.82$.

In [10] we find that the quality of synchronization increases rapidly for $c>0.4$ and is largest for the open loop configuration. The quality of synchronization drops fast when the mismatch of the delay time is larger than a few percent. Since the open loop configuration with $c=1$ does not have the problem of delay time mismatch and shows the highest quality synchronization, $c=1$ is the preferred configuration for chaotic synchronization and communication with this delayed optoelectronic feedback system.

\section{CHAOS COMMUNICATION}

The achievement of synchronization of two chaotic semiconductor lasers with optoelectronic feedback allows us to employ communication schemes in which the message is put into the transmitter dynamics via a reversible operation, e.g. addition or multiplication. The transmitted signal is then a combination of the message and the chaotic output of the transmitter. Since on the receiver side we know the expected chaotic output of the transmitter, due to the identical synchronization, we can use the expected output to extract the message from the received signal. In [5] we evaluated numerically the performance of a multiplicative communication scheme and found favorable bit error rates for a wide range of the $c$ factor. Here we report the result of an experiment in which the reversible function is addition [11].

The schematic experimental setup is shown in Fig. 4, where we use the open loop configuration $c=1$. It is important to note that the message signal $\mathrm{m}(\mathrm{t})$ enters the dynamics of the transmitter via the feedback loop. Therefore the combined signal of output plus message enters symmetrically both the transmitter and receiver, which ensures that synchronization is maintained when we transmit information.

Fig. 5 shows the results of an encoding/decoding experiment of narrow pulses with $100 \mathrm{MHz}$ repetition rate. The time series of the pulse encoding/decoding is shown in Fig. 5. The top trace is the received signal with the message encoded. The second trace is the local receiver laser output, which is due to the synchronization equal to the transmitter output before the addition of the message. The message is therefore recovered by subtracting the receiver output (second trace) from the received signal 


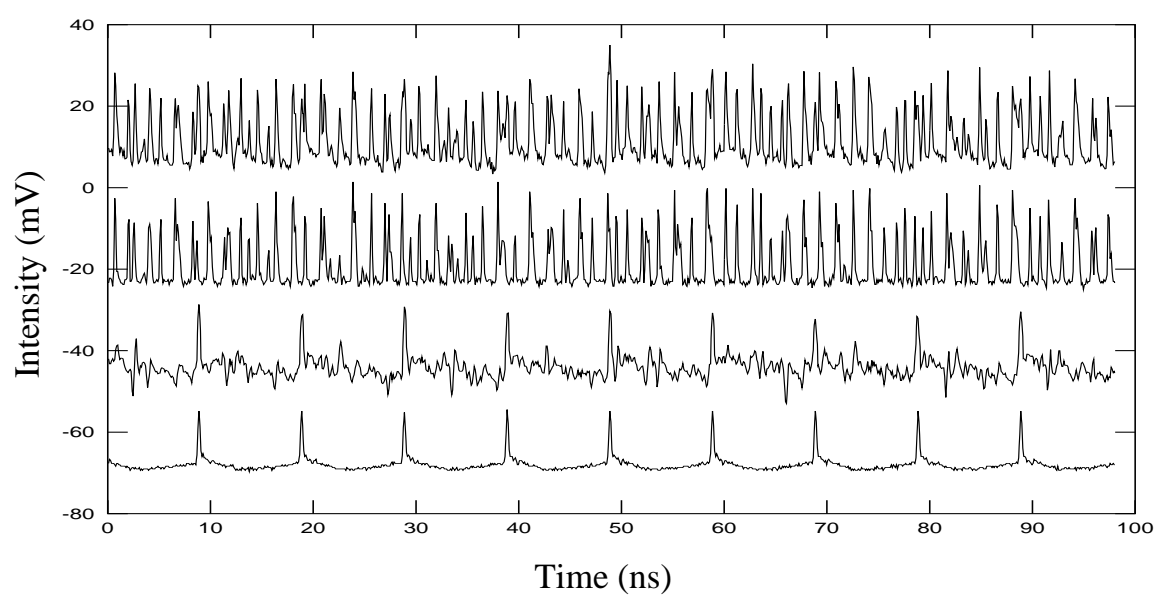

FIGURE 5. Encoding/decoding of digital pulses with $100 \mathrm{MHz}$ repetition rate. Time series of received signal (top), receiver laser output (middle), recovered digital message (third), and original message (bottom).

(top trace). The recovered message shows good quality of decoding as indicated by the recovered pulse train in the third trace, which is to be compared with the original 'message', which is a set of periodic pulses, in the bottom trace.

In conclusion, we demonstrated that optoelectronic feedback lasers exhibit high frequency chaotic oscillations and enter chaos via a quasi-periodic route. We achieved chaos synchronization of two such systems. We furthermore demonstrated that a highspeed pulsing message can be successfully encoded and decoded. Since the transmitter and receiver remain synchronized when a message is transmitted the bit rate of the encoded message can be possibly as high as the repetition rate of the chaotic pulsing, which is about $600 \mathrm{MHz}$ at present time. With even faster components the bit rate can be improved to multigiga bits per second.

\section{REFERENCES}

1. Goedgebuer,J. P., Larger, L. and Porte, H., Phys. Rev. Lett., 80, 2249-2252, (1998).

2. VanWiggeren, G. D., and Roy, R., Phys. Rev. Lett., 81, 3547-3550, (1998).

3. Abarbanel, H. D. I., Kennel, M. B., Buhl, M., and Lewis, C., Phys. Rev. A, 60, 2360-2374, (1999).

4. Tang, S. and Liu, J. M., IEEE J. Quantum Electron., 37, 329-36, (2001).

5. Abarbanel, H. D. I., Kennel, M. B., Illing, L., Tang, S., Chen, H. F., and Liu, J. M. "Synchronization and communication using semiconductor lasers with optoelectronic feedback", submitted to IEEE J. Quantum Electron., (2001).

6. Grigorieva, E. V., Haken, H., and Kaschenko, S. A., Optics Comm., 165, 279-292, (1999).

7. Pieroux, D., Erneux, T., Luzyanina, T., and Engelborghs, K., Phys. Rev. E, 63, 036211-1 to -11, (2001).

8. Abarbanel, H. D. I. and Kennel, M. B., Phys Rev. Lett., 80, 3153-3156, (1998)

9. Lewis, C., Abarbanel, H. D. I., Kennel, M. B., Buhl, M., and Illing, L., Phys. Rev. E, 63, 016215-1 to $-15,(2000)$

10. Tang, S. and Liu, J. M., Optics Lett., 26, 596-598, (2001)

11. Tang, S. and Liu, J. M.,"Message encoding/decoding using chaotic pulsing semiconductor lasers," OFC 2001 Technical Digest Series, Anaheim, 2001,paper WDD46, pp. WDD46-1 to -3. 